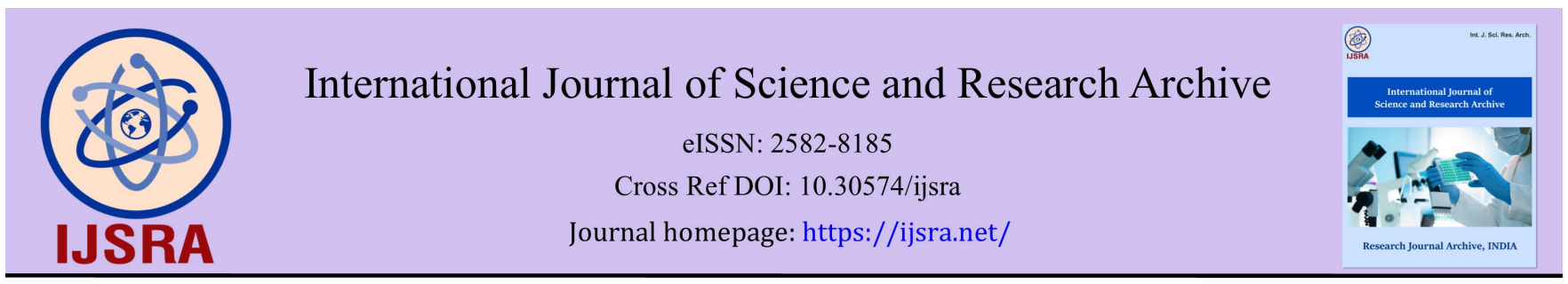

(RESEARCH ARTicle)

Check for updates

\title{
Evaluation of genotoxicity of ethanolic extracts of Platostoma palustre by micronucleus assay
}

Yu-Hsing Lin 1, \#, Yun-Xuan Chang 2, \#, Tzu-Yun Chi 2, \#, Hsiao-Yun Chen 2, \#, Ying-Ching Hung 2, \#, Chia-Yu Lin 2, Guan-Hong Chen 2, Ya-Peng Wang 2, Ping-Min Huang 2, Tsung-Han Wu 2, Yen-Jung Lu 2, Chien-Chao Chiu ${ }^{2}$, Ching-Feng Chiu ${ }^{3}$, Hsuan-Wen Chiu ${ }^{4}$, Wei-Huang Tsai ${ }^{5}$, Chia-Chi Chen ${ }^{2}$ and Shao-Wen Hung 2, 6,*

${ }^{1}$ Bachelor Degree Program in Pet Healthcare, Yuanpei University of Medical Technology, Xiangshan, Hsinchu 300, Taiwan.

2 Division of Animal Industry, Animal Technology Research Center, Agricultural Technology Research Institute, Xiangshan, Hsinchu 300, Taiwan.

${ }^{3}$ Graduate Institute of Metabolism and Obesity Sciences, College of Nutrition, Taipei Medical University, Taipei 110, Taiwan.

${ }^{4}$ Department of Biotechnology and Bioindustry Sciences, College of Bioscience and Biotechnology, National Cheng Kung University, Tainan 701, Taiwan.

${ }^{5}$ Department of Science and Technology, Council of Agriculture, Executive Yuan, Taipei 100, Taiwan.

${ }^{6}$ Department of Nursing, Yuanpei University of Medical Technology, Hsinchu 300, Taiwan.

* Contributed equally to this work.

International Journal of Science and Research Archive, 2022, 05(01), 133-139

Publication history: Received on 18 January 2022; revised on 23 February 2022; accepted on 25 February 2022

Article DOI: https://doi.org/10.30574/ijsra.2022.5.1.0051

\begin{abstract}
Platostoma palustre $(P p)$ jelly is a traditional food. $P p$ has been used as folk medicine and is effective against heat-shock, hypertension and diabetes. Therefore, the aim of this study was to evaluate the ethanolic extracts of $P p^{\prime}$ genotoxicity. The ethanolic extracts of $P p$ by using $40 \%$ ethanol for extraction. Evaluation of genotoxicity of ethanolic extracts of Pp by micronucleus assay was performed in vivo. During the in vivo genotoxicity-evaluated experiment, the experimental animal's clinical behavior, body weight (BW), food consumption, and the percentage of RET/RBCs (reticulocytes/red blood cells) and MN-RET/RETs (micronucleated reticulocytes/reticulocytes) after the treatments of $P p$ ethanolic extracts were evaluated. Both sexes Institute of Cancer Research (ICR) mice were given three daily treatments by intraperitoneal injection of $2 \mathrm{mg} / \mathrm{kg}$ of mitomycin C (genotoxicity induction) or by oral route of $200 \mu \mathrm{L}$ of PBS (normal control group). Until $48 \mathrm{~h}$ after the last treatment, K2-EDTA-anticoagulated peripheral blood specimens were collected. These blood samples were processed for the microscopy-based analysis using Giemsa stain and the percentage of reticulocytes and micronucleated reticulocytes was determined. The results were shown that the experimental animal's clinical behaviors were normal in all groups. The BW and food consumption were no significant difference between all groups. RET/RBCs (\%o) in male or female ICR mice in the negative control group, the normal control group, the high dose of $P p$ ethanolic extract group, the middle dose of $P p$ ethanolic extract group, and the low dose of $P p$ ethanolic extract group were respectively $7.8 \pm 0.8 / 8.6 \pm 0.8,23.2 \pm 1.5 / 22.1 \pm 1.3,22.8 \pm 1.6 / 22.1 \pm 1.7,23.2 \pm 1.5 / 22.6 \pm$ 1.0 and $22.2 \pm 1.9$ / $23.9 \pm 1.9$; MN-RET/RETs (\%) in male or female ICR mice in the negative control group, the normal control group, the high dose of $P p$ ethanolic extract group, the middle dose of $P p$ ethanolic extract group, and the low dose of $P p$ ethanolic extract group were $2.0 \pm 0.0 / 2.0 \pm 0.0,43.2 \pm 10.6 / 39.6 \pm 10.9,1.8 \pm 0.4 / 1.6 \pm 0.5,1.6 \pm 0.5 /$ $1.4 \pm 0.5$, and $1.8 \pm 0.4 / 1.6 \pm 0.5$, respectively. Both RET/RBCs (\%o) and MN-RET/RETs (\%o) in male or female ICR mice in the negative control group were significantly difference than the other groups $(\mathrm{p}<0.001)$. Taken all results together, $P p$ ethanolic extracts were without genotoxicity. Therefore, $P p$ ethanolic extracts were safety.
\end{abstract}

Keywords: Ethanolic extraction; Genotoxicity; In vivo; Micronucleus assay; Reticulocytes; Platostoma palustre extracts

\footnotetext{
* Corresponding author: Shao-Wen Hung

Division of Animal Industry, Animal Technology Research Center, Agricultural Technology Research Institute, Xiangshan, Hsinchu 300, Taiwan.
} 


\section{Introduction}

In recent years, factors such as changes in people's health concepts, changes in living habits, emphasis on dietary intake, the promotion of alternative medical concepts, and advances in life science and technology have promoted the vigorous development of today's health food industry. Due to the gradual aging of the world and the rising market demand in Association of Southeast Asian Nations (ASEAN) countries, it is estimated that the functional food market is promising. Platostoma palustre $(P p)$ is an annual plant that is mainly distributed in tropical and subtropical regions, including Taiwan, Indonesia, Vietnam, southern China, and Burma [1-6]. The related food with $P p$ as tea, herbal jelly, and sweet soup with herbal jelly are popular during the summer. Additionally, the heated herbal jelly with $P p$ is admired by many Taiwanese in winter. $P p$ has been used as folk medicine. $P p$ has been verified that possessed many functional compounds such as polysaccharides (gum) with a unique aroma and texture, sterol compounds, stigmasterol, $\alpha$-sitosterol, tripterpene compounds, oleanolic acid, volatile compounds (caryophyllene oxide, $\alpha$-caryophyllene, eugenol, benzene acetaldehyde, and 2,3-butanedione etc), essential oil (n-hexadecanoic acid, linoleic acid, and linolenic acid), volatile oil (chavibetol, n-hexadecanoic acid, and $\alpha$-cadinol), and ursolic acid [7-10]. Therefore, theses functional compounds of Platostoma palustre have indicated that many biological effects are effective against and attenuating the metabolic syndrome, heat-shock, hypertension, diabetes, liver disease, muscle and/or joint pains, hyperglycemia, inflammation, oxidant activity, free radical scavenging effects, acute and chronic hepatitis, and caner growth [11-13]. However, whether the safety of $P p$ should be evaluated, especially genotoxicity.

According to the research and investigation, Taiwan's agricultural science papers are cited more than other fields, showing Taiwan's agricultural science and technology has R\&D energy that cannot be ignored. Recently, the industrialization of scientific and technological achievements has attracted the attention of the government and the public. At the same time, the demonstration of this global trend is also an opportunity for Taiwan's agricultural science and technology to once again demonstrate the miracle. Looking at the industrialization development of agricultural science and technology in Taiwan today, a considerable number of resources and technologies have been accumulated in upstream R\&D, but how to quickly transform technologies and resources into commodities through the industrialization development platform, and then form settlements and industries, is a topic worthy of attention. Therefore, it is an urgent need to establish a stable, accurate and rapid animal test evaluation system to evaluate the safety of healthy foods and functional foods as a pre-market screening tool [14-19].

An increasing number of reports are shown that "functional foods" are containing some functional component benefits may be observed to enhance short-term well-being and increasingly considered healthful. Based on these findings, the study was aimed to upgrade the production capacity of high value-added agricultural products. For the quality assurance, toxicity assessment for the functional foods is necessary. There is an emerging requirement to apply in vivo genotoxicity assays to evaluate the carcinogenic potential of these functional foods. Based on these requirements, we suggested that the mammalian erythrocyte micronucleus assay is used for the detection of damage induced by the test substance to the chromosomes of erythroblasts [20-23]. In this study, we tried to institute a sensitive and stable method for quantifying the formation of micronuclei in erythrocytes in vivo. The objective of this study was to evaluate the safety of $P p$ ethanolic extracts via micronucleus assay in vivo. The resulting data will enable us to evaluate the potential toxicity assessment of agricultural functional products in the future.

\section{Material and methods}

\subsection{Chemicals and Reagents}

Mitomycin C (Cayman, CAS 50-07-7, Item No.11435), Giemsa staining solution kit (Baso corporation, WG0-020), phosphate-buffered saline (PBS; Sigma-Aldrich, Cat. No. P3813), saline (Taiwan Biotech Co., LTD, Cat. No. 100-1201101), Zoletil 50 (Virbac, Carros, France), and DPX mountant (Sigma-Aldrich, Cat. No. 44581).

\subsection{Source of Dried Platostoma palustre}

The dried $P p$ were ordered from the traditional markets in Miaoli, Taiwan.

\subsection{0\% Ethanolic Extraction}


10-15 g samples of $P p$ powder were soaked for $24 \mathrm{~h}$ at room temperature with $200 \mathrm{~mL}$ of $40 \%$ ethanol, followed by filtration through Whatman \#1 filter paper. The filtrates were then evaporated in vacuo to dryness and weighed to determine the yields.

\subsection{Preparation of Mitomycin C}

Weighing $0.75 \mathrm{mg}$ of mitomycin $\mathrm{C}$ and add it to $3 \mathrm{~mL}$ of saline and mix it to a concentration of $250 \mu \mathrm{g} / \mathrm{mL} \mathrm{mitomycin} \mathrm{C.}$ To draw mitomycin $\mathrm{C}$ solution [2 $\mathrm{mg} / \mathrm{kg}$ body weight (BW)] with a $1 \mathrm{~mL}$ syringe for the subsequent intraperitoneal injection (the injection volume is adjusted according to the $\mathrm{BW}$ of mice).

\subsection{Experimental Animals and Experimental Design}

Adult male and female ICR mice [6 weeks old; 50 mice; BW between 26-27 g)] with specific pathogen-free conditions were used for this study, were purchased from BioLASCO Taiwan Co., Ltd. (Yilan, Taiwan). These ICR mice were fed with standard laboratory diet (No. 5001, LabDiet ${ }^{\circledR}$; PMI Nutrition International, St. Louis, MO, USA) and distilled water ad libitum during the experimental period. The environment was maintained room temperature $\left(24-27^{\circ} \mathrm{C}\right)$ and $60 \%-70 \%$ humidity with a photoperiod of 12-hr light/12-hr dark cycle. The study will begin after a week acclimation. The Institutional Animal Care and Use Committee (IACUC) of Agricultural Technology Research Institute inspected all animal experiments and this study comply with the guidelines of protocol IACUC-110045 approved by the IACUC ethics committee. The 25 ICR male mice and 25 ICR female mice were divided respectively into as the normal control group ( $\mathrm{n}=10 ; 5$ male and 5 female mice), the negative control group ( $\mathrm{n}=10 ; 5$ male and 5 female mice), the high dose $(1,000$ $\mathrm{mg} / \mathrm{kg} \mathrm{BW}$ ) of $P p$ ethanolic extract group ( $=10 ; 5$ male and 5 female mice), the middle dose $(500 \mathrm{mg} / \mathrm{kg} \mathrm{BW})$ of $P p$ ethanolic extract group ( $\mathrm{n}=10 ; 5$ male and 5 female mice), and the low dose (250 mg/kg BW) of $P p$ ethanolic extract group ( $\mathrm{n}=10 ; 5$ male and 5 female mice). The mitomycin $\mathrm{C}$-induced genotoxicity were performed in the negative control group. The clinical behaviors, BW, food consumption, and blood smear exanimation were monitored and performed during the experiment.

\subsection{Collection of Peripheral Blood from ICR Mice}

The ICR mice were anesthetized with Zoletil 50 and the blood collection site was cleaned with $70 \%$ alcohol. The blood was stored in anticoagulant tubes containing $\mathrm{K}_{2}$-EDTA for use in the subsequent experiments. ICR mice in three doses of $P p$ ethanolic extract groups were respectively administered three doses of $P p$ ethanolic extracts (the interval between each time must exceed 24 hours), and peripheral blood in all groups must be collected at $48^{\text {th }}$ hours after the last administration of $P p$ ethanolic extracts.

\subsection{Preparation of Blood Smear and Giemsa Staining with Light Microscopic Examination}

Blood smear on the glass slides were prepared by a senior researcher. The air-dried blood smears were stained with 1 $\mathrm{mL}$ of Giemsa stain for 1-3 minutes, then add $2 \mathrm{~mL}$ of PBS (pH 7.2) or distilled water for 2-6 minutes. The slides were washed with the running water, air-dried and mounted by DPX mountant, and then placed under a light microscope $(1,000 \times$ magnification) to observe and count the number of cells. At least, 1,000 reticulocytes were observed in each ICR mouse, the number of micronuclei was recorded, and the proportion of reticulocytes in total red blood cells was calculated.

\subsection{Statistical Analysis}

The data were expressed as mean \pm SD. All comparisons were made by one-way ANOVA and all significant differences are reported at ${ }^{* * *} p<0.001$.

\section{Results}

\subsection{Change of Mouse's BW in All Groups}

The ethanolic extracts of $P p$ by using $40 \%$ ethanol for extraction were provide in the oral administration in the three $P p$ ethanolic extract groups. In this study, the clinical behavior observation indexes of mice in each group were normal during the experiments. During the experiments, the mice in each group had smooth hair, normal hair color, and the normal activity. Moreover, all mice were survival until the end of the experiments. The survival rate was $100 \%$ (50/50) (data not shown). The BW of mice was detected on day 0 , day 7 , day 8 , day 9 , and day 10 . The day 0 was mice entering the animal room. The day 7 to day 9 were genotoxicity induction. The day 9 to day 10 were three doses of $P p$ ethanolic extract administration. During the experiments, the mice's BW continued to rise and there was no statistically significant difference in BW between all groups (Figure 1). 


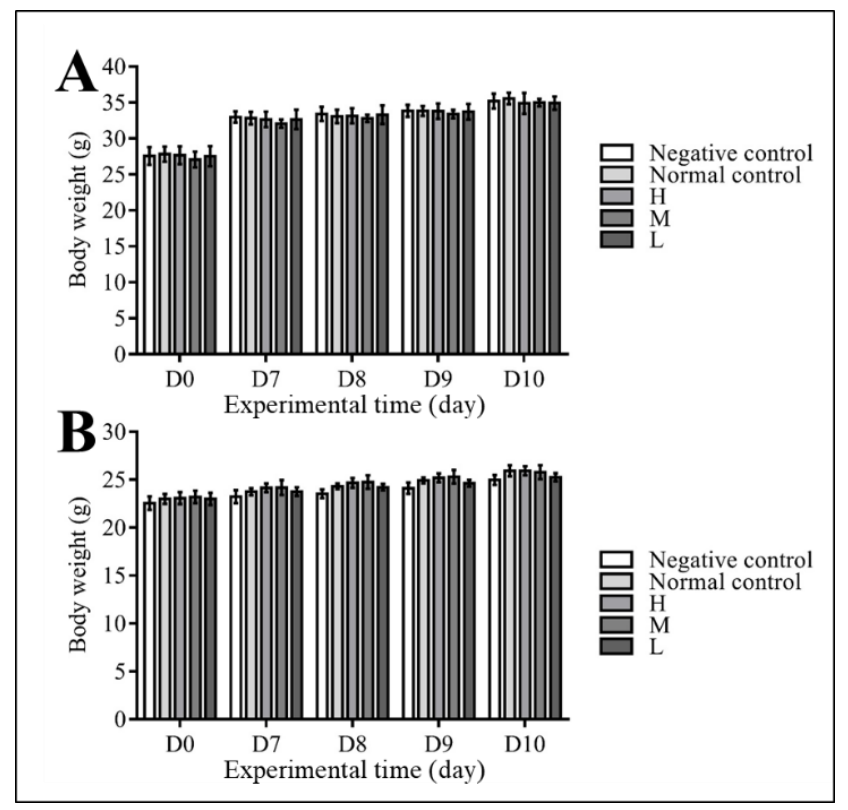

Figure 1 Change of body weight before and after the treatments of three doses of Platostoma palustre ethanolic extracts. (A) Male ICR mice. (B) Female ICR mice. All data are expressed as mean \pm SD. ' $H$ ' is high dose $(1,000 \mathrm{mg} / \mathrm{kg} \mathrm{BW})$ of Platostoma palustre ethanolic extract group. ' $\mathrm{M}$ ' is middle dose (500 mg/kg BW) of Platostoma palustre ethanolic extract group. 'L' is low dose $(250 \mathrm{mg} / \mathrm{kg} \mathrm{BW})$ of Platostoma palustre ethanolic extract group

\subsection{Change of Mouse's Food Consumption in All Groups}

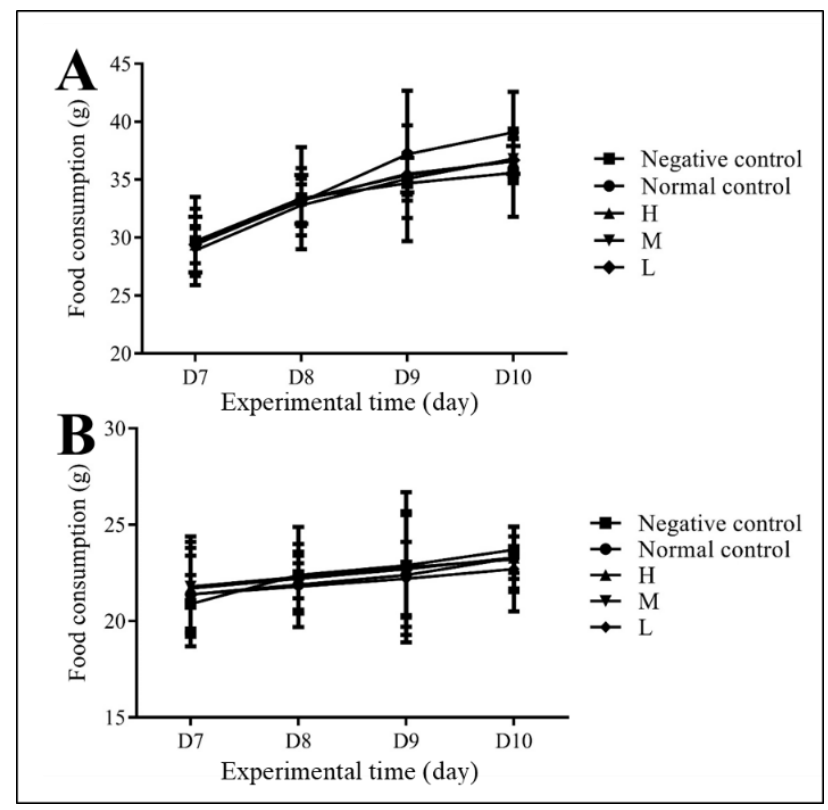

Figure 2 Change of food consumption after the treatments of three doses of Platostoma palustre ethanolic extracts. (A) Male ICR mice. (B) Female ICR mice. All data are expressed as mean \pm SD. 'H' is high dose $(1,000 \mathrm{mg} / \mathrm{kg} \mathrm{BW})$ of Platostoma palustre ethanolic extract group. 'M' is middle dose (500 mg/kg BW) of Platostoma palustre ethanolic extract group. 'L' is low dose ( $250 \mathrm{mg} / \mathrm{kg} \mathrm{BW}$ ) of Platostoma palustre ethanolic extract group

In this study, the mouse's food consumption in each group were monitored during the experiments. The food consumption of mice was detected on day 0 , day 7 , day 8 , day 9 , and day 10 . The day 0 was mice entering the animal room? The day 7 to day 9 were genotoxicity induction. The day 9 to day 10 were three doses of $P p$ ethanolic extract administration. During the experiments, three doses of Pp ethanolic extracts did not cause a decrease in appetite and abrupt decrease in food intake (Figure 2). 


\subsection{Change of RET/RBCs and MN-RET/RETs Percentages in All Groups}

Mice' peripheral anticoagulant blood were collected at 48 hours after the third administration, respectively. Blood samples were prepared for blood smears and processed with Giemsa staining. After blood sample processing, they were examined by using light microscope. The percentages of RET/RBCs and MN-RET/RETs were evaluated. The results showed that the male and female mice in the negative control group were induced genotoxicity. The percentage of RET/RBCs in the negative control group was significantly decrease than the other groups $(p<0.001)$ (Figure 3A-B). Except for the negative control group, there were no significant difference for the comparison of the other groups $(p>$ 0.05) (Figure 3A-B). RET/RBCs (\%o) in male or female ICR mice in the negative control group, the normal control group, the high dose of $P p$ ethanolic extract group, the middle dose of $P p$ ethanolic extract group, and the low dose of $P p$ ethanolic extract group were respectively $7.8 \pm 0.8 / 8.6 \pm 0.8,23.2 \pm 1.5 / 22.1 \pm 1.3,22.8 \pm 1.6 / 22.1 \pm 1.7,23.2 \pm 1.5$

/ $22.6 \pm 1.0$, and $22.2 \pm 1.9 / 23.9 \pm 1.9$ (Figure 3A-B). The percentage of MN-RET/RETs in the negative control group was significantly increase than the other groups $(p<0.001)$ (Figure 3C-D). Except for the negative control group, there were no significant difference for the comparison of the other groups $(p>0.05)$ (Figure 3C-D). MN-RET/RETs (\%o) in male or female ICR mice in the negative control group, the normal control group, the high dose of $P p$ ethanolic extract group, the middle dose of $P p$ ethanolic extract group, and the low dose of $P p$ ethanolic extract group were $2.0 \pm 0.0 /$

$2.0 \pm 0.0,43.2 \pm 10.6 / 39.6 \pm 10.9,1.8 \pm 0.4$ / $1.6 \pm 0.5,1.6 \pm 0.5 / 1.4 \pm 0.5$, and $1.8 \pm 0.4$ / 1.6 \pm 0.5 , respectively (Figure 3C-D). Taken all results together, $P p$ ethanolic extracts were without genotoxicity. Therefore, $P p$ ethanolic extracts were safety.

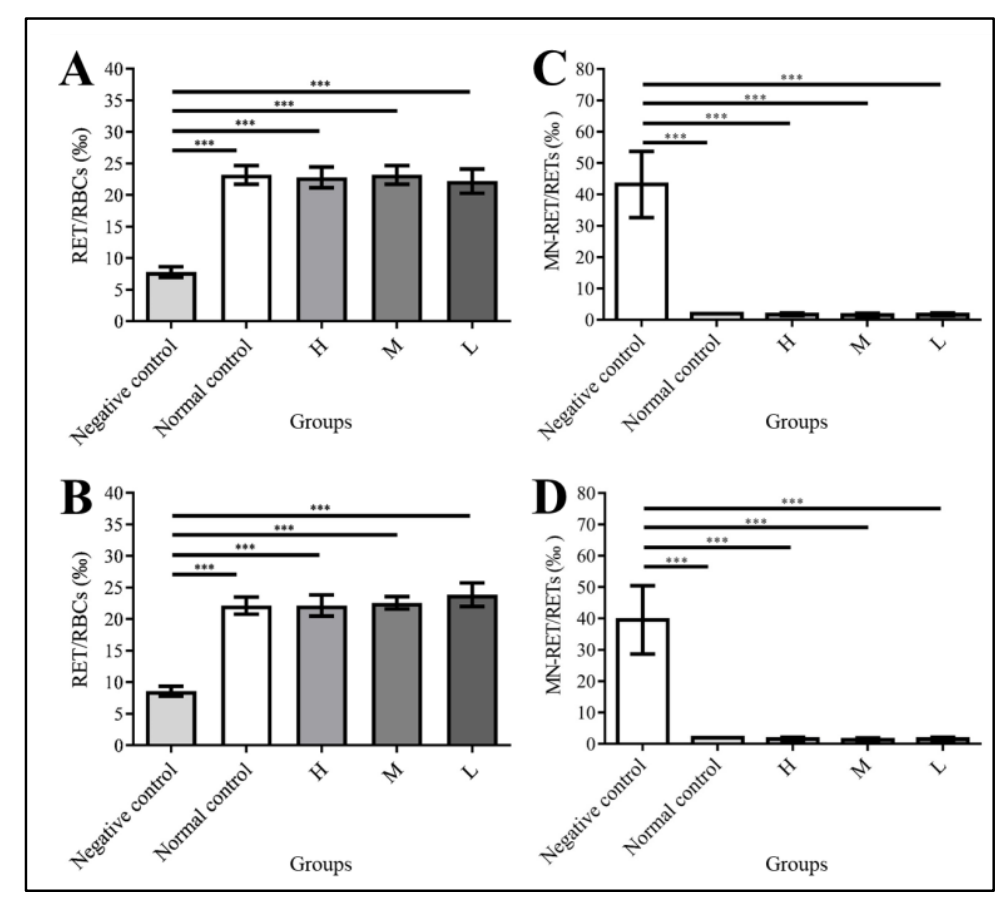

Figure 3 Change of RET/RBCs and MN-RET/RETs after the treatments of three doses of Platostoma palustre ethanolic extracts. (A) RET/RBCs (\%) in male ICR mice. (B) RET/RBCs (\%o) in female ICR mice. (C) MN-RET/RETs (\%o) in male ICR mice. (D) MN-RET/RETs (\%) in female ICR mice. ' $\mathrm{H}$ ' is high dose $(1,000 \mathrm{mg} / \mathrm{kg} \mathrm{BW})$ of Platostoma palustre ethanolic extract group. 'M' is middle dose (500 mg/kg BW) of Platostoma palustre ethanolic extract group. 'L' is low dose (250 $\mathrm{mg} / \mathrm{kg} \mathrm{BW}$ ) of Platostoma palustre ethanolic extract group. All data are expressed as mean \pm SD. All significant differences compared to negative control group were reported at $* * * p<0.001$

\section{Discussion}

In order to evaluate the safety of health food, Taiwan government has formulated a method for evaluating the safety of health food [28]. Among health food safety assessment methods, genotoxicity test methods can be divided into in vivo and in vitro tests, the purpose of which is to detect the genetic damage and extent directly or indirectly caused by the test substances, including the microbial gene mutation assays, the in vitro genotoxicity analysis of mammalian cells, and the genotoxicity analysis of animals in vivo [24-26]. In vivo genotoxicity assays in animals generally were used chromosomal damage assays of rodent hematopoietic cells, including bone marrow cell micronucleus assays, 
chromosomal abnormalities assays or peripheral blood micronucleus assays. Due to the high sensitivity of the micronucleus assays, this method is the most commonly used method for testing toxic drug-induced chromosomal aberrations in vivo [27].

Previously, the effect of different ethanolic concentrations on antioxidant properties and cytoprotective activities of $P p$ has been verified. The antioxidant activities of Mesona procumbens ethanolic extracts which displayed variable antioxidant levels. The $60 \%$ Mesona procumbens ethanolic extracts exhibited higher antioxidant activities that possessed a protective capability for the biological membrane system to prevent and treat oxidative stress-related disorders [11-13]. According to our previous data, the bio-functional components and concentrations of $P p$ ethanolic extracts via $90 \%$ ethanol extraction were chlorogenic acid $(0.30 \mathrm{mg} / \mathrm{mg} P p$ ethanolic extracts $)$, caffeic acid $(0.70 \mathrm{mg} / \mathrm{mg}$ $P p$ ethanolic extracts), rutin $(0.80 \mathrm{mg} / \mathrm{mg} P p$ ethanolic extracts), astragaloside IV (7.50 mg/mg $P p$ ethanolic extracts), and rosmarinic acid $(15.90 \mathrm{mg} / \mathrm{mg} P p$ ethanolic extracts). Among of these bio-functional components of $P p$ ethanolic extracts via $90 \%$ ethanol extraction, the contents of astragaloside IV and rosmarinic acid were higher than others. Many literatures have been demonstrated that astragal side IV and rosmarinic acid possessed anti-tumor abilities. In this study, $P p$ ethanolic extracts via $40 \%$ ethanol extraction were used. Therefore, we want to understand the functional effects of $P p$ ethanolic extracts and their component concentrations.

\section{Conclusion}

The purpose of this study was to induce genotoxicity in ICR mice with mitomycin $\mathrm{C}$ and evaluate whether $P p$ ethanolic extracts were safe. Taken these results together, we successfully established the phenomenon of micronucleus in peripheral blood cells induced by mitomycin C in ICR mice. After detecting via this genotoxicity mouse platform, we have demonstrated that $P p$ ethanolic extracts were no genotoxicity. In the future, we hope this genotoxicity mouse platform will be provided to detect the genotoxicity in the test samples.

\section{Compliance with ethical standards}

\section{Acknowledgments}

All authors thank the Council of Agriculture in Taiwan (Executive Yuan) [grant numbers 110 AS-14.3.2-ST-a2] for supporting this study.

\section{Disclosure of conflict of interest}

The authors declare no conflict of interest.

\section{Statement of ethical approval}

The Institutional Animal Care and Use Committee (IACUC) of Agricultural Technology Research Institute inspected all animal experiments and this study comply with the guidelines of protocol IACUC-110045 approved by the IACUC ethics committee.

\section{References}

[1] Hsieh Y, Lin SP, Wu L, Fang W, Hwang TS. Effects of Antrodia camphorata extracts on anti-oxidation, antimutagenesis and protection of DNA against hydroxyl radical damage. BMC Complement Altern. Med. 2015; 15: 237.

[2] Huang DJ, Chen HJ, Lin CD, Lin YH. Antioxidant and antiproliferative activities of water spinach (Ipomoea aquatica Forsk) constituents. Bot Bull Acad. Sin. 2005; 46: 99-106.

[3] Pervin M, Hasnat MA, Lee YM, Kim DH, Jo JE, Lim BO. Antioxidant activity and acetylcholinesterase inhibition of grape skin anthocyanin (GSA). Molecules. 2014; 19: 9403-9418.

[4] Pourmorad F, Hosseinimehr SJ, Shahabimajd N. 2006. Antioxidant activity, phenol and flavonoid contents of some selected Iranian medicinal plants. Afr. J. Biotechnol. 2006; 5: 1142-1145.

[5] Yen GC, Chen HY. Antioxidant activity of various tea extracts in relation to their antimutagenicity. J .Agric. Food. Chem. 1995; 43: 1455-1461. 
[6] Widyaningsih TD, Adilaras P. Hepatoprotective effect of extract of black Cincau (Mesona palustris BL) on paracetamol-induced liver toxicity in rats. Adv. J. Food Sci. Technol. 2013; 5: 1390-1394.

[7] Ahmed MB, Khater MR. Evaluation of the protective potential of Ambrosia maritima extract on acetaminopheninduced liver damage. J. Ethnopharmacol. 2001; 75: 169-174.

[8] Bessems JG, Vermeulen NP. Acetaminophen (acetaminophen) -induced toxicity: molecular and biochemical mechanisms, analogues and protective approaches. Crit. Rev. Toxicol. 2001; 31: 55-138.

[9] Kumar A, Sanjiv S, Chandel S. Evaluation of hepatoprotective activity of Abelmoschus moschatus seed in paracetamol induced hepatotoxicity on rat. J. Pharmacy. 2012; 2: 43-50.

[10] Okawa M, Kinjo J, Nohara T, Ono M. DPPH (1, 1-Diphenyl-2-Picrylhydrazyl) radical scavenging activity of flavonoids obtained from some medicinal plants. Boil. Pharm. Bull. 2001; 24: 1202-1205.

[11] Widyaningsih T, Sukardiman D. Djoko AP, Win D. Immunomodulatory effects of the water extract of black Cincau (Mesona palustris BL) against interferon gamma expression, immunosurveillance activation and apoptosis on benzo (a) pyrene-induced fibrosarcoma carcinogenesis in mice. J. Technol. Food Ind. 2012; 23: 29-35.

[12] Yen GC, Hung CY. Effects of alkaline and heat treatment on antioxidative activity and total phenolic of extracts from Hsian-tsao (Mesona procumbens Hemsl.). Food Res. Int. 2001; 33: 487-492.

[13] Yen GC, Yeh CT, Chen YT. Protective effect of Mesona procumbens against tert-butyl hydroperoxide-induced acute hepatic damage in rats. J. Agric. Food Chem. 2004; 52: 4121-4127.

[14] Bryce SM, Bemis JC, Avlasevich SL, Dertinger SD. In vitro micronucleus assay scored by flow cytometry provides a comprehensive evaluation of cytogenetic damage and cytotoxicity. Mutat. Res. 2007; 630: 78-91.

[15] Hara M, Nakagawa S, Fujioka E, Ayukawa E. Detection of micronuclei in peripheral blood of mitomycin C-treated mice using supravital staining with acridine orange. Mutat. Res. 1992; 278: 175-179.

[16] Recio L. Comparison of flow cytometry- and microscopy-based methods for measuring micronucleated reticulocyte frequencies in rodents treated with nongenotoxic and genotoxic chemicals. Mutat. Res. 2008; 649: 101-113.

[17] Tometsko AM, Torous DK, Dertinger SD. Analysis of micronucleated cells by flow cytometry. 1. Achieving high resolution with a malaria model. Mutat. Res. 1993a; 292: 129-135.

[18] Tometsko AM, Dertinger SD, Torous DK. 1993b. Analysis of micronucleated cells by flow cytometry. 2. Evaluating the accuracy of high-speed scoring. Mutat. Res. 1993b; 292: 137-143.

[19] Witt KL, Livanos E, Kissling GE, Torous DK, Caspary W, Tice RR, Tometsko AM, Dertinger SD, Torous DK. Analysis of micronucleated cells by flow cytometry. 2. Evaluating the accuracy of high-speed scoring. Mutat. Res. 1993; 292: 137-143.

[20] Garriott ML, Piper CE, Kokkino AJ. A simplified protocol for the mouse bone marrow micronucleus assay. J. Appl. Toxicol. 1988; 8: 141-144.

[21] Sato S, Inui N, Ikeda Y, Hiraga Y. A comparison of intraperitoneal injection and oral gavage in the micronucleus test with mitomycin C in mice. Mutat. Res. 1989; 223: 387-390.

[22] Hayashi M, MacGregor JT, Gatehouse DG, Adler I-D, Blakey DH, Dertinger SD, Krishna G, Morita T, Russo A, Sutou S. In vivo rodent erythrocyte micronucleus assay. II. Some aspects of protocol design including repeated treatments, integration with toxicity testing, and automated scoring. Environ. Mol. Mutagen. 2000; 35: 234 -252.

[23] Hayashi M, Tice RR, MacGregor JT, Anderson D, Blakey DH, Kirsh-Volders M, Oleson FBJr, Pacchierotti F, Romagna F, Shimada H, Sutou S, Vannier B. In vivo rodent erythrocyte micronucleus assay. Mutat. Res. 1994; 312: $293-304$.

[24] OE G, Karp NA, Ng BL, Jackson SP, Adams DJ, McIntyre RE. A high -throughput in vivo micronucleus assay for genome instability screening in mice. Nat. Protoc. 2015; 10: 205-215.

[25] Krishna G, Hayashi M. In vivo rodent micronucleus assay: protocol, conduct and data interpretation. Mutat. Res. 2000; 455: 155-166.

[26] Mavournin KH, Blakey DH, Cimino MC, Salamone MF, Heddle JA. The in vivo micronucleus assay in mammalian bone marrow and peripheral blood. Mutat. Res. 1990; 239: 29-80.

[27] Lin JW, Chen CC, Chiu CC, Yang CY, Hung SW. A study to establish a mouse genotoxicity model by mitomycin C induction. J. Chin. Soc. Anim. Sci. 2017; 46: 193-207. 